\title{
Shadow Detection and Compensation in Aerial Images using MATLAB
}

\author{
Sachin Tiwari \\ M.Tech. Scholar in Deptt. of \\ ECE SAGAR Institute of \\ Research and Technology \\ Bhopal M.P. India
}

\author{
Krishna Chauhan \\ Assistant Professor in Deptt. of \\ ECE SAGAR Institute \\ Research and of Technology \\ Bhopal M.P. India
}

\author{
Yashwant Kurmi \\ Research scholar in Deptt. of \\ ECE Maulana Azad National of \\ Technology Bhopal M.P. India
}

\begin{abstract}
Recently, Kuo-Liang Chung presented an efficient algorithm uses the ratio of hue over intensity as the parameter to determine the coarse shadow map and then the local thresholding method (STS) in which it needs the different threshold levels which is comparatively more time consuming (and if the threshold is not proper then the resultant image is not the desired image, because threshold determination is typical task in itself) for fine shadow determination in color aerial images. In our proposed method we again modify the ratio with some empirical relation that give better result taking less time. Under four testing images, experimental results show that, for all four images that are low and medium intensity images have better shadow detection but the shadow compensation algorithm is gives the good result in all the testing images. We also have the comparison between three algorithms Tsai's, STS and our proposed algorithms with result of shadow detected images [15].
\end{abstract}

\section{Keywords}

Shadow compensation, modified hue intensity ratio, shadow detection.

\section{INTRODUCTION}

In this modern era everything is being watched out by cameras (all time watchman) in the absence of paid watchman. This camera provides the security at every moment. At present high resolution cameras have ability to detect the things very accurately. In the images that have shadow it is difficult to identify the object or thing. Shadows can either aid or confound scene interpretation, depending on whether we model the shadows or ignore them. In addition, shadows are also responsible to degrade the image quality. Shadows in images are typically affected by several phenomena in the scene, including physical phenomena such as lighting conditions, type and behaviour of shadowed surfaces, occluding objects; etc .Human vision system is very immune to shadows. We do not find any difficulty in recognizing, tracking objects even with shadows. But in the case of computer vision, shadows create problems and reduce the reliability of the system. In addition, shadows are also responsible to degrade the image quality. Therefore, shadow compensation is an important pre-processing step for computer vision and image enhancement algorithm [1], [2], [3].Shadow compensation from image can be used for object detection, such as cancer detection, military object detection etc., as sometimes images are covered by shadows. After compensating these shadows, objects in the images will appear more obviously so that they are recognized correctly. Therefore, shadow detection and compensation is an important pre-processing step for computer vision and image enhancement. Shadow compensation from respective image can also be used for object detection, in medical field image analysis, in military object detection, in satellite image analysis etc. Many times all type of images are covered by shadows. After compensating these shadows, objects in the images will appear more clearly so that they are recognized correctly.

\subsection{Shadow Identification}

V.J.D.Tsai. Presented an efficient algorithm to detect shadows for color aerial RGB [4], [13], [16] images. The input image can be first transformed into the HSI; Hue, saturation and value (HSV); luma, blue-difference chroma and red-difference chroma $(\mathrm{YCbCr})$, hue, chroma and value (HCV) or luminance, hue and saturation (YIQ) color models. Tsai first calculated the ratio of the hue over intensity for each pixel to construct the ratio map and then a global threshold of a constructed ratio map is determined to identify shadows [7], [14]. To improve the performance we have done some modification in that ratio using some empirical relations.

1.2 Shadow removal: The shadow compensation problem can be broken down into two main constituent parts: shadow identification and shadow compensation. Shadow identification is a well-defined concept, in which the presence of shadows upon different material image surfaces is detected and their exact position upon those surfaces is located. We primarily focus on shadow compensation upon any one of these material surfaces. We assume that such an identification method has recognized a surface as having a shadow on it and passed it to our algorithm. Our shadow compensation approach is based on a simple shadow model where lighting consists of directed light and environment light [8].

\section{PROPOSED WORK}

First take the Image as input then apply the median filter to remove the noise components. And the following equation is given to transform the RGB color model into the HSI color model.

$$
\begin{aligned}
& {\left[\begin{array}{l}
I \\
V_{1} \\
V_{2}
\end{array}\right]=\left[\begin{array}{ccc}
\frac{1}{3} & \frac{1}{3} & \frac{1}{3} \\
\frac{-\sqrt{6}}{6} & \frac{\sqrt{6}}{6} & \frac{\sqrt{6}}{3} \\
\frac{1}{6} & \frac{-2}{\sqrt{6}} & 0
\end{array}\right]\left[\begin{array}{l}
R \\
G \\
B
\end{array}\right]} \\
& I_{e}=\frac{1}{3} R+\frac{1}{3} G+\frac{1}{3} B \\
& \mathrm{H}_{\mathrm{e}}=\left(\tan ^{-1}\left(\frac{\mathrm{V}_{1}}{\mathrm{~V}_{2}}\right)+\pi\right) \times \frac{255}{2 \pi}
\end{aligned}
$$

Where $V_{1}$ and $V_{2}$ have been defined in (1) and value of $I_{e}$ and $\mathrm{H}_{\mathrm{e}}$ are bounded in range $[0,255]$. 
2.1 Shadow Identification To detect shadows, we must consider the appearance of the local and surrounding regions. Shadowed regions tend to be dark, with little texture, but some non-shadowed regions may have similar characteristics. Surrounding regions that correspond to the same material can provide much stronger evidence. Based on HSI color model, the intensity-equivalent Image $I_{e}$ and the hue-equivalent Image $\mathrm{H}_{\mathrm{e}}$ used in the modified ratio map. Here we take the hue over intensity ratio with some modification.

$$
\mathrm{R} 1=(\mathrm{H}+1) /(\mathrm{I}+0.1)
$$

Now to vary the pixel range we use this ratio as exponential

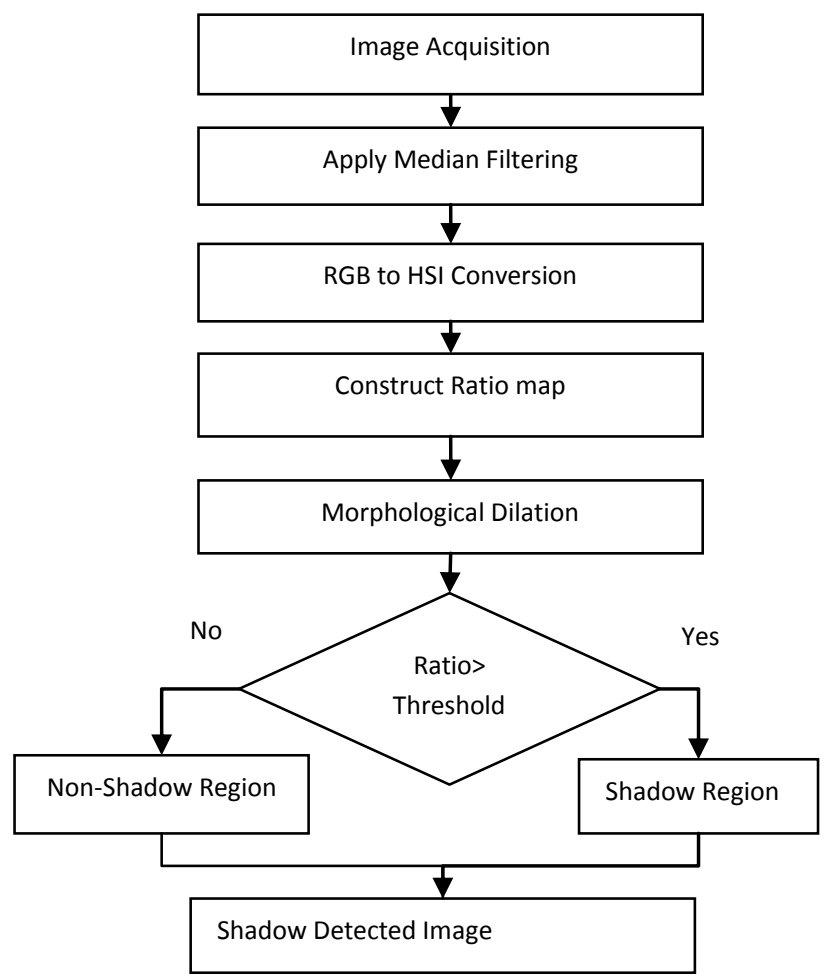

Figure: (I) Flow chart and Algorithm for Shadow Detection

power empirical values 0.5 to 0.7 . It will provide the ratio as

$$
\begin{aligned}
& \mathrm{B} 1=0.5^{\wedge}(\mathrm{R} 1) \\
& \mathrm{B} 3=0.7^{\wedge}(\mathrm{R} 1)
\end{aligned}
$$

The result of Tsai's is just the ratio image of Hue over Intensity there is not a clear boundary between shadow and non-shadow region and STS method giving the result that is not satisfactory for the top part of eaves at mid- right corner of the House in figure (II). It is shows as a non shadow region for shadowed part. But our modified ratio gives the better result detecting the shadow with greater accuracy.

2.2 Shadow Compensation: Thus, to eliminate the shadow from an image (I) can be done by a pixel-wise product of I by a scaling factor image $b$ such that

$$
I_{c}=b I
$$

Where $I_{c}$ the shadow compensated image and $b$ scales the light illumination of the image to eliminate shadow. The proposed algorithm is applicable to a single-colour channel that is, red, green or blue. This implies a separate $b$ for each channel so it can be assumed that compensating shadow from a colour image can be done by implementing the proposed method separately for each colour channel. In addition choosing $\mathrm{b}$ is not trivial, because, in many situations, the illumination information is coupled with complex textural information. Figure (II) shows the flow chart and algorithm for shadow compensation. Figure (VI) shows the original image of House image of house along with Shadow detected Image and respective shadow compensated Image. The result in above, image of house showing very good some bright spot of very small size are there but they are not like to affect the shadow detection. This ratio provides the detection process as the coarse shadow detection as compared to previous existing methods in less time of process.

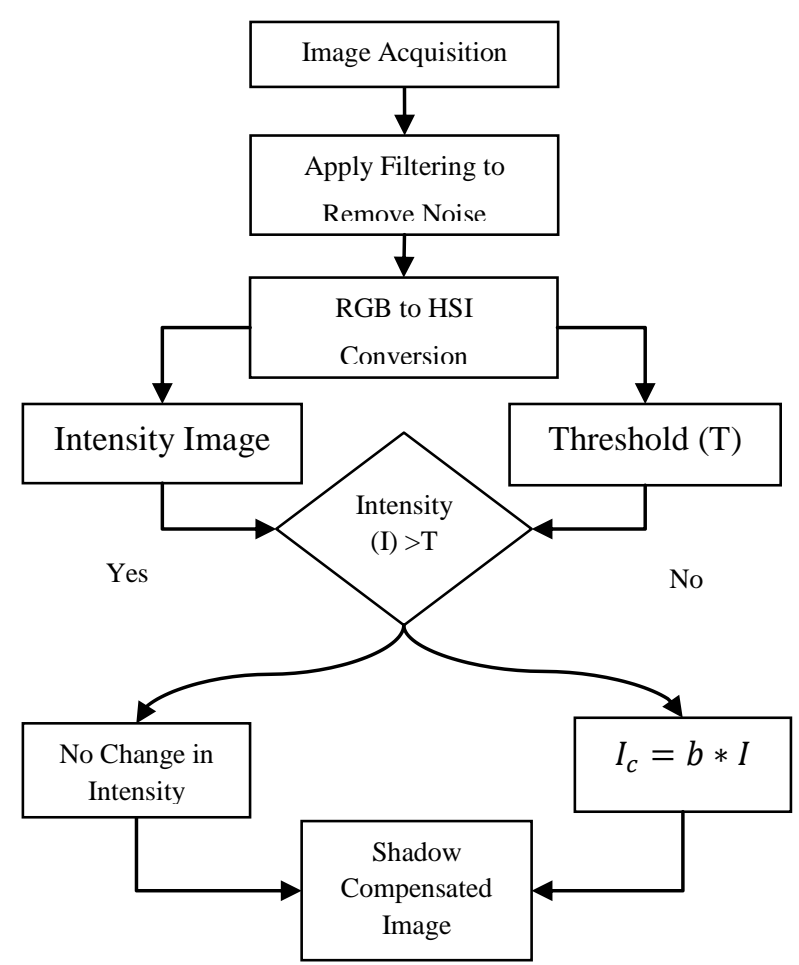

Figure: (II) Flow chart and Algorithm for Shadow Compensation

After determining the ratio we replace the shadowed pixels (below threshold) with negative value of max pixel intensity then perform. The threshold is calculated by using Otsu's thresholding Method [12]. Dilation operation and shadow detected image is found out. This method is the simplest form of shadow detection

\section{PERFORMANCE PARAMETERS:}

The evaluation matrices used in Tsai's model are adopted to evaluate the accuracy of the concerned shadow detection algorithms. Based on the concept of the error matrices [15], [16] and terminologies defined in [17], [18] three types of accuracy, namely the producers accuracy, the users accuracy and the overall accuracy, are used in objective evaluation.

The three types of accuracy are described as follows. The first type of accuracy is the producers accuracy, which contain two parameters $\mathrm{P}_{\mathrm{S}}$ and $\mathrm{P}_{\mathrm{N}}$ and they are defined by

$$
\text { Producer's accuracy: } \begin{aligned}
\mathrm{P}_{\mathrm{S}} & =\frac{\mathrm{TP}}{\mathrm{TP}+\mathrm{FN}} \\
\mathrm{P}_{\mathrm{N}} & =\frac{\mathrm{TN}}{\mathrm{FP}+\mathrm{TN}}
\end{aligned}
$$


where true positive (TP) denotes the number of true shadow pixels which are identified correctly; false negative (FN) denotes the number of true shadow pixels which are identified as non-shadow pixels; false positive (FP) denotes the number of non-shadow pixels which are identified as true shadow pixels; and true negative (TN) is the number of non-shadow pixels which are identified correctly. The parameter $\mathrm{P}_{\mathrm{S}}\left(\mathrm{P}_{\mathrm{N}}\right)$ denotes the ratio of the number of correctly detected true shadow (non-shadow ) pixels over that of total true shadow (non-shadow) pixels. The second type accuracy is the user's accuracy in terms of $A_{S}$ and $A_{N}$ which are defined as

$$
\begin{array}{ll}
\text { User's accuracy: } & \mathrm{A}_{\mathrm{S}}=\frac{\mathrm{TP}}{\mathrm{TP}+\mathrm{FP}} \\
& \mathrm{A}_{\mathrm{N}}=\frac{\mathrm{TN}}{\mathrm{TN}+\mathrm{FN}}
\end{array}
$$

The parameter $A_{S}\left(A_{N}\right)$ denotes the ratio of the number of correctly detected true shadow (non-shadow) pixels over that of the total detected true shadow (non-shadow) pixels and thus the user's accuracy can be used to measure the precision of the shadow detection algorithm. Combining the accuracies of user and the producer, the third type of accuracy $\tau$ defined as follows can be used to evaluate the correctness percentage [15] of the algorithm:

$$
\text { Overall accuracy } \quad \tau=\frac{\mathrm{TP}+\mathrm{TN}}{\mathrm{TP}+\mathrm{TN}+\mathrm{FP}+\mathrm{FN}}
$$

Where TP + TN denotes the number of correctly detected true shadow and non-shadow pixels; $\mathrm{TP}+\mathrm{TN}+\mathrm{FP}+\mathrm{FN}$ is equal to the number of total pixels in the image. Among these metrics, the producer's accuracies are measures of the correctness of the algorithm and indicate how well pixels of known categories are correctly classified. The user's accuracies are measures of the precision of the algorithm and indicate the probabilities of pixels been correctly classified into actual categories on the ground. The overall accuracy is a measure of relative effectiveness of the algorithm and reports the percentage correct or the closeness of the estimate to the true value. These metrics together demonstrate the significance of a shadow detection algorithm. For a good algorithm, values of these evaluation metrics should be high.

The Table I for image in figure (II) shows the producer's Accuracy, User's Accuracy and overall Accuracy.

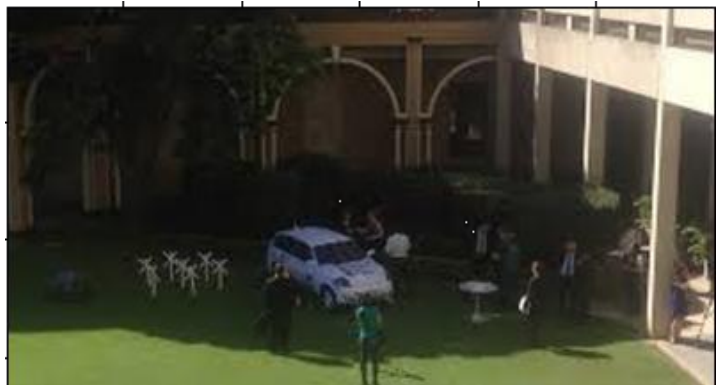

(a)

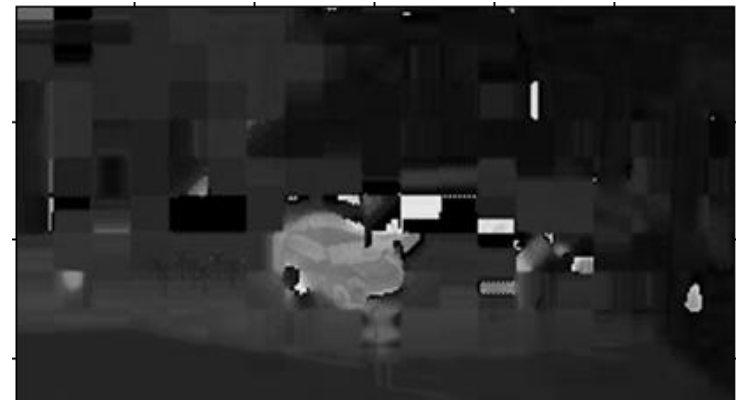

(b)

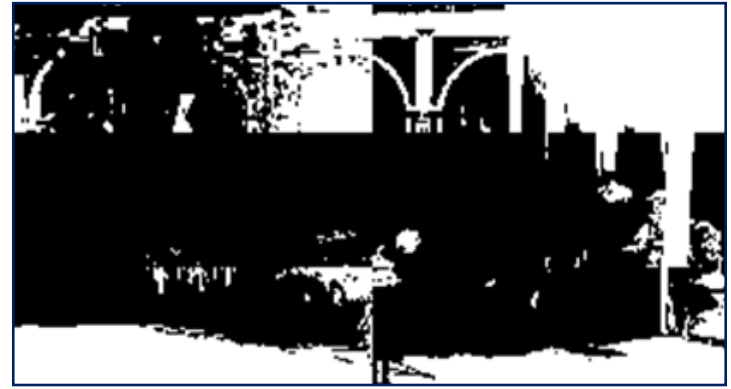

(c)

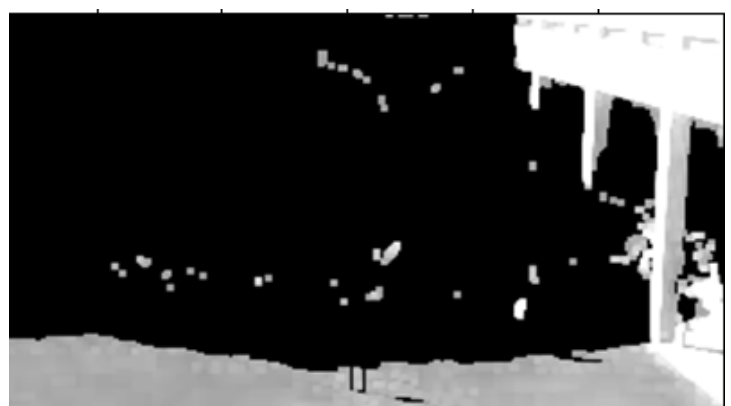

(d)

Figure: (III) Comparison of Shadow Detected Images sing Different Algorithms (a) Original Image of House, (b) Shadow Detected Image of Tsai Model, (c) Shadow Detected Image using STS Method (d) Shadow Detected Image Using Our Modified Ratio of Hue over Intensity.

\section{RESULTS ANALYSIS}

The algorithm proposed here will remove shadows from an image surface without prior knowledge of the shadow location upon that surface. The proposed method is based on determining the illumination profile of the image surface, including shadow and fully illuminated areas.

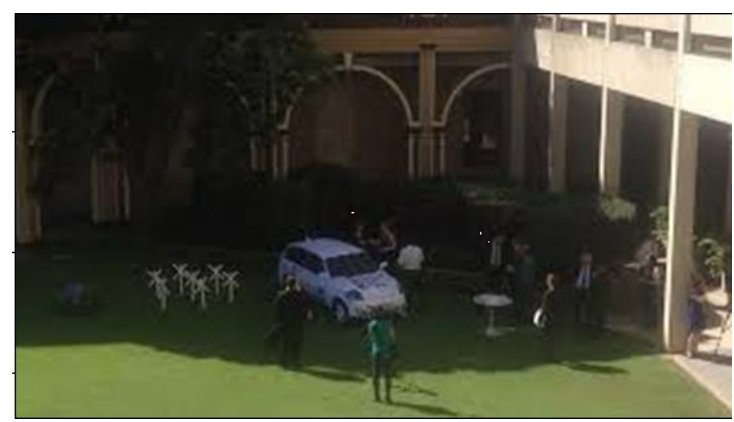

(a)

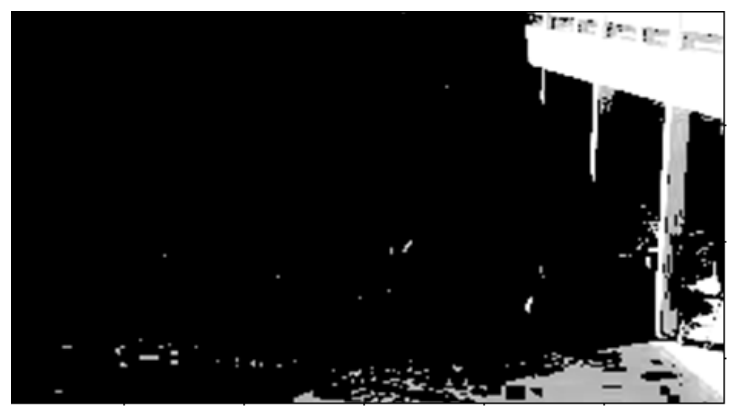

(b) 


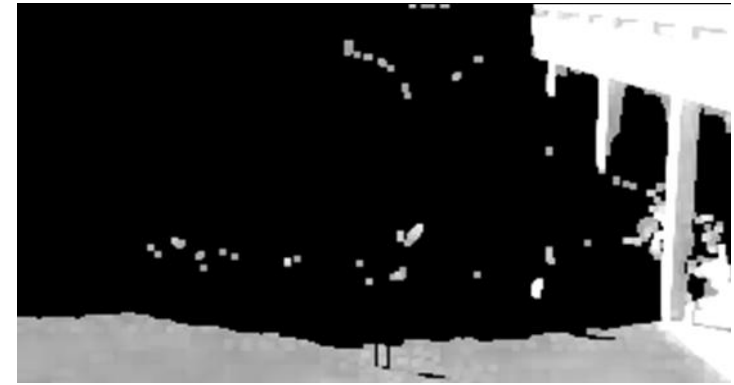

(c)

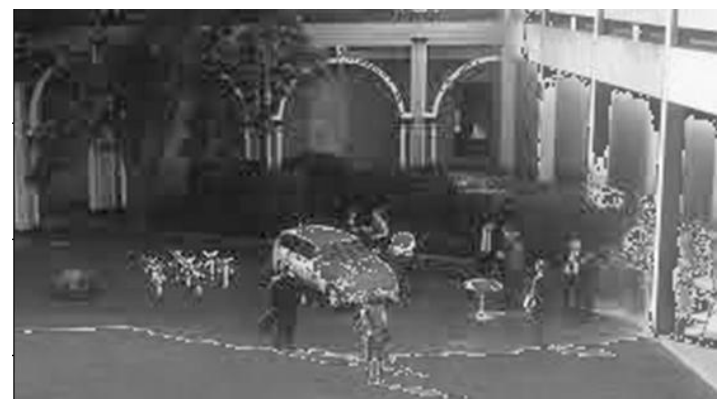

(d)

Figure: (IV) (a) Original Image of House, (b) Shadow Detected Image, (c) Shadow Detected Image after Dilation and (d) Shadow Compensated Image by Applying Intensity Variation Method.

\section{CONCLUSION AND FUTURE SCOPE}

Our results show advancement on the existing state-of-the-art methods, particularly in the algorithms ability to complex and compound shadow arrangements, which fall beyond the scope of many existing automatic shadow compensation algorithms. We have made no prior assumptions about the location, form or even the existence of the umbra or penumbra, and hence, the algorithm is highly robust to many forms of shadow. In the proposed method, the shadow is compensated from textured surfaces and there is little discernible texture degradation in the overall image.

TABLE I

Shadow Detection Accuracy for Image of House

\begin{tabular}{|c|c|c|c|c|c|}
\hline \multirow{2}{*}{ Method } & \multicolumn{2}{|c|}{$\begin{array}{c}\text { Producer's } \\
\text { accuracy }\end{array}$} & \multicolumn{2}{c|}{$\begin{array}{c}\text { User's } \\
\text { Accuracy }\end{array}$} & $\begin{array}{c}\text { Overall } \\
\text { Accuracy }\end{array}$ \\
\cline { 2 - 6 } & $\begin{array}{c}\text { PS } \\
(\%)\end{array}$ & $\begin{array}{c}\text { PN } \\
(\%)\end{array}$ & $\begin{array}{c}\text { AS } \\
(\%)\end{array}$ & $\begin{array}{c}\text { AN } \\
(\%)\end{array}$ & $\tau(\%)$ \\
\hline Proposed & $\mathbf{9 7 . 5 0}$ & $\mathbf{9 2 . 8 7}$ & $\mathbf{8 6 . 3 3}$ & $\mathbf{8 7 . 3 0}$ & $\mathbf{8 6 . 8 2}$ \\
\hline Tsai's & $\mathbf{2 9 . 4 5}$ & $\mathbf{3 1 . 5 1}$ & $\mathbf{5 0 . 8 6}$ & $\mathbf{7 1 . 4 2}$ & $\mathbf{6 1 . 1 4}$ \\
\hline $\begin{array}{c}\text { Kuo- } \\
\text { Liang } \\
\text { Chung }\end{array}$ & $\mathbf{2 9 . 4 5}$ & $\mathbf{3 1 . 5 1}$ & $\mathbf{5 8 . 5 8}$ & $\mathbf{7 9 . 0 8}$ & $\mathbf{6 8 . 8 3}$ \\
\hline
\end{tabular}

The major assumption in the proposed method is that textural details are smaller than shadow details. Further study in discriminating textural edges from shadow edges, for scenes that do not meet this criterion, may be of merit. Finally, we should say something about processing overhead. The algorithm was implemented in MATLAB ${ }^{\circledR}$ as a test implementation. It currently takes several minutes to process an image; however, we hope to better this in future work.
Future Scope The shadow detection and compensation is very much required to identify the object in images in tough whether such as fog, heavy rain and snow fall the satellite images can be used to make efficient for accessing the image data. The process of shadow compensation invariably involves dealing with the shadow boundary. This necessitates distinguishing between shadow edges and reflectance edges. Reflectance edges that cross or coincide with shadow edges (whether sharp shadow edges or wide penumbra) must be restored consistently with the same type of reflectance edges outside the shadow. This difficulty is especially significant for algorithms that work in the gradient domain since the gradients in such edges are composed of both reflectance and shadow changes [10], thus requiring the algorithm to modify only the shadow term of the shadow edge gradient.

\section{REFERENCES}

[1] C. Fredembach, G. Finlayson,: Simple shadow removal", In Proceedings of International Conference on Pattern Recognition, (ICPR), pp. 832-835, 2006

[2] J.M. Wang, Y.C. Chung, C.L. Chang, S.W. Chen, "Shadow Detection and Removal for Traffic Images", Proc. IEEE International Conference on Networking, Sensing and Control, volume 1, pp. 649-654, 2004.

[3] T. Chen, W. Yin, X.S. Zhou, D. Comaniciu, and T.S. Huang, "Illumination Normalization for Face Recognition and Uneven Background Correction Using Total Variation Based Image Models", Proceedings CVPR, volume 2, pp. 532-539, 2005.

[4] V.J.D. Tsai, " A comparative study on shadow removal of color aerial images in invariant color models," IEEE Trans .Geosci. Remote sens.,vol.44 ,no.6 ,pp 1661-1671, jun.2006

[5] Kuo-Liang Chung "Efficient Shadow Detection Of Color Aerial Images Based on Successive Thresholding Scheme," IEEE Trans. on Geosciences and Remote Sensing, Vol. 47, No. 2 Feb 2009.

[6] Kumar, S.; Pant, M.; Ray, A. "Differential evolution embedded Otsu's method for optimized image thresholding" , Information and Communication Technologies (WICT), 2011 World Congress, Page(s): 325 - 329, Year: 2011.

[7] G.Z.yang, D.N.Firmin, P.Burger, and S.R.Underwood "Structure Adaptive Anisotropic image filtering, "Image Vis. Comput., vol.14. no.2pp.135-145,Mar.1996.

[8] R. McFeely C. Hughes* E. Jones M. Glavin: "Removal of non-uniform complex and compound Shadows from textured surfaces using adaptive Directional smoothing and the thin plate model" Published in IET Image Processing Received on 5th August 2009. Revised on 8th April 2010.

[9] Barrow, H.G., Tenenbaum, J.M.: 'Recovering intrinsic scene characteristics from images'. Proc. Computer Vision Systems, 1978, pp. 3-26.

[10] L. Xu, F. Qi, and R. Jiang, "Shadow Removal from a Single Image," Proc. IEEE Int'l Conf. Intelligent Systems Design and Applications, pp. 1049-1054, 2006.

[11] W.K.Pratt, "Digital Image Processing, 2nd ed. New York: Wiley. 
[12] N. Otsu's, "A Threshold selection method from gray level histograms," IEEE Trans. Syst., Man, Cybern.,vol.SMC-9 ,no.1, pp.62-69, jan.1979.

[13] R. C. Gonzalez R.E. Woods, Digital Image Processing, 2nd ed. reading, MA:Addison-Wesley,2002.

[14] Kuo-Liang Chung, Yi-Ru Lin, and Yong-Huai Huang "Efficient Shadow Detection of Color Aerial Images Based on Successive Thresholding Scheme " IEEE Transactions On Geosciences And Remote Sensing, Vol. 47, No. 2, February 2009.

[15] Eli Arbel and Hagit Hel-Or. "Shadow Removal Using Intensity Surfaces and Texture Anchor Points". IEEE. transactions on pattern analysis and machine intelligence, vol. 33, no. 6, June 2011.

[16] Rafael C.Gonzalez, Richerd E. Woods and Steven L. Eddins "Digital Image Processing Using MATLAB®" 2nd ed. Tata Mc Graw Hill.

[17] Zhang Jin-Yu Chen Yan Huan Xian-Xiang,"Edge detection of images based on improved Sobel operator and Genatic Algorithm,".

[18] J.Yao and Z. Zhang, "Systematic static shadow detection," in Proc. 17th Int. Conf. pattern Recog. 2004, vol. 2, pp 76-79. 